Zu der vorstehenden Tabelle ist noch folgendes zu bemerken: Bei den Kakaos der Firmen Schaal und Reichardt (Monarch) finden sich Zahlen für freies Theobromin, die weit über dem Durchschnitt stehen. Es sind das Zahlen, die erhalten wurden, als ich durch erhöhte Zugabe von Alkoholat versuchte, eine höhere Ausbeute an Theobromin zu erzielen als früher (vergl. S. 580). Bei diesen Zahlen handelt es sich aber, wie schon oben ausgeführt wurde, nicht um reines Theobromin; sie sind nur der Vollständigkeit halber mit aufgenommen.

Ferner fällt auf, daß bei dem Stollwerk'schen und dem Suchard'schen Kakao die Zahlen der Einzelbestimmungen für beide Formen des Theobromins nicht miteinander übereinstimmen. Der Grund hierfür ist, daß ich bei diesen Bestimmungen keinen Wert mehr darauf gelegt habe, die beiden Formen quantitativ zu trennen. Es lag mir hier mehr daran, zu sehen, ob die Endergebnisse noch übereinstimmen, wenn auch nicht alles freie Theobromin quantitativ mit dem Alkoholat herausgelöst war, bevor die Behandlung mit verdünnter Schwefelsäure vorgenommen wurde. Das ist aber der Fall, und es ist daher für die Praxis eine Trennung nicht nötig. Man wird zweifellos ebenso gute Ergebnisse erhalten, wenn man zunächst nur das Fett bestimmt, wie ich es früher vorgeschlagen habe, und dana in dem Rückstande das gesamte Theobromin in einer Operation, indem man ihn direkt der Behandlung mit verdünnter Schwefelsäure unterwirft und weiter verarbeitet, wie ich es oben beschrieben habe.

Wie die Verhältnisse bei der rohen Bohne liegen, soll noch Gegenstand einer weiteren Untersuchung sein. Ebenso sind Arbeiten zur Gewinnung und Untersuchung des Glykosides bereits in Angriff genommen.

\title{
Die Fettbestimmung in Schokolade mittels Chloralalkoholats.
}

\author{
Von \\ Ad. Kreutz. \\ Mitteilung aus dem Pharmazeutischen Institut der Universitat Straf- \\ burg i. Els. \\ [Eingegangen am 12. September 1908.]
}

Vor einiger Zeit habe ich in dieser Zeitschrift ${ }^{1}$ ) über eine Methode der Fettbestimmung im Kakao mittels Chloralalkoholats berichtet und damals schon angedeutet, daß Versuche zur Übertragung der Methede auf Schokolade bereits in Angriff genommen seien. Im folgenden sollen die Ergebnisse dieser Versuche mitgeteilt werden.

Es war nicht von vornherein anzunehmen, daß das Verfahren ohne weiteres auf Schokoladen übertragbar sei, da Schokolade ja außer Kakao noch eine Reihe anderer Substanzen enthält. Wie diese Zusätze - Zucker, Milch, Gewürze etc. sich gegen ätherische Lösungen des Chloralalkoholates verhalten, war aber noch nicht untersucht. Fethaltige Zusätze — wie bei den Milchschokoladen - können kein

1) Diese Zeitschrift 1908, 15, 680. 
Hindernis für die Anwendbarkeit des neuen Verfahrens bilden, da alle Fette mit Chloralalkoholat leicht in Lösung zu bringen sind, Anorganische Körper - wie die Mineralbestandteile der Milch, Gewürze etc. - sind aber vollkommen unlöslich in ätherischen Lösungen des Alkoholates, wie schon $\mathrm{Mauch}^{1}$ ) nachgewiesen hat. Sie bilden also ebenfalls kein Hindernis für das Verfahren. Unbekannt ist aber, wie sich die verschiedenen Zuckerarten, die Extraktivstoffe der Gewürze etc. verhalten. Alle in Betracht kommenden Substanzen auf ihre etwaige Löslichkeit in Chloralalkoholat zu prüfen, würde aber zu weit gehen und den Rahmen dieser Arbeit weit überschreiten. Ich habe es daher vorgezogen, die Methode zunächst unverändert in Anwendung zu bringen, wie ich sie früher beschrieben habe, und zu untersuchen, ob außer Fett noch andere Stoffe in Lösung geben. Als Versuchsobjekt diente eine Milchschokolade Sarotti. Das ätherische Filtrat, das außer dem Alkoholate nur das Fett enthalten sollte, war absolut klar und leicht gelb gefärbt. Als dann aber der Äther und das Alkoholat abdestilliert worden waren, hinterblieb im Kolben außer dem Fett noch eine feste harte, bräunliche Masse, die in den gebräuchlichen Fetllösungsmitteln -- Äther, Petroläther, Chloroform, Tetrachlorkohlenstoff - unlöslich war. Das aus diesem Rückstande extrahierte und vom Lösungsmittel befreite Fett war hell und schmolz klar.

Bei dieser Milchschokolade waren die braunen festen Rückstände an feuchter Luft zerfließlich. Sie bildeten nach kurzem Stehen im Kolben Tropfen; die auf dem Filter befindlichen Anteile waren von dem Papier aufgesogen. Bei anderen Schokoladen, z. B. der von Felsche, zeigte sich diese Zerfließlichkeit nicht in so ausgesprochenem Maße. Löslich in Wasser waren diese Rückstände aber immer. Von einer näheren Untersuchung habe ich vorläufig abgesehen; es scheinen aber Kohlenhydrate vorzuliegen, da die wässerigen Lösungen in allen Fällen Feh ling'sche Lösung sofort reduzierten.

Wenn auch die Mengen dieses festen Rückstandes gering waren, so ließ sich seine Bildung in dem fetthaltigen Filtrate doch auf keine Weise verhindern. Die Fettbestimmung in Schokoladen nach der Chloralalkoholat-Methode gestaltet sich daher nach den Erfahrungen längerer Versuchsreihen folgendermaßen:

2-3 g Schokolade werden, in grobe Stücke gebrochen, in einem ErlenmeyerKolben abgewogen. Dazu gibt man $3 \mathrm{~g}$ festes Chloralalkoholat und bringt den Kolben auf ein siedendes Wasserbad. Das Alkoholat schmilzt bald und nun läßt sich die weich gewordene Schokolade leicht mit dem Alkoholate zu einem dünnen Brei anrühren. Nach etwa 15 Minuten nimmt man den Kolben vom Wasserbade und zieht die Schmelze mit Äther aus, wie ich es früher für die Untersuchung von Kakao beschrieben habe, $\mathrm{E}$ s empfiehlt sich, recht dichte Filter anzuwenden, da sonst leicht ein trübes Filtrat erhalten wird, aus dem sich dann rasch ein hellbrauner Niederschlag absetzt, der beim Abdestillieren des Äthers zu fortgesetztem heftigen Stoßen Veranlassung gibt. Man filtriert in einen Fraktionskolben; aus dem Filtrat destilliert man zunächst den Äther ab. Das Alkoholat entfernt man am raschesten durch Destillation unter vermindertem Druck, Die Vorlage darf dabei nicht gekühlt werden, da sonst durch Krystallisation leicht eine Verstopfung des Destillationsrohres eintritt. Die Destillation geht bei einer Wasserbadtemperatur von $75^{\circ}$ rasch von statten und ist in längstens einer halben Stunde beendet.

1) M a u ch, Inaug.-Dissertation, Straß3burg i. Els. 1898. 
Der im Kolben verbleibende Rückstand besteht alsdann aus dem Fett und den vorher genannten braunen Anteilen. Mit Tetrachlorkohlenstoff wird nun in der Kälte das Fett gelöst und durch Filtration von dem unlöslichen Teil getrennt. Nach dem Verjagen des Tetrachlorkohlenstoffes bleibt das Fett vollkoinmen klar geschmolzen zurück. Die letzten Reste des Lösungsmittels müssen im Trockenschranke bei 100 bis $105^{\circ}$ entfernt werden. Die nachstehende Tabelle enthält die Ergebnisse der Untersuchung von 3 Schokoladen:

\begin{tabular}{|c|c|c|}
\hline \multirow[b]{2}{*}{ Bezeichnung der Schokolade } & \multicolumn{2}{|c|}{ Fettgehalt } \\
\hline & $\begin{array}{c}\text { nach der } \\
\text { Chloralmethode }\end{array}$ & $\begin{array}{l}\text { mit Äther } \\
\text { extrahiert }\end{array}$ \\
\hline $\begin{array}{l}\text { Mileh Schokolade Sarotti } \\
\text { Feine Vanille-Schokolade von W. Felsche in Leipzig-Gohlis } \\
\text { Chokolat Suchard zum Rohessen } . . .\end{array}$ & $\begin{array}{c}\% / 0 \\
28,85 ; 28,93 \\
18,61 ; 18,38 \\
29,16 ; 29,22\end{array}$ & $\begin{array}{l}\% \\
29,00 \\
18,80 \\
29,17\end{array}$ \\
\hline
\end{tabular}

Die Zahlen zeigen, daß die Ergebnisse unter sich und auch mit den nach dem Ätherextraktionsverfahren erhaltenen gut übereinstimmen. Die Chloral-Methode läßt sich also auch bei der Fettbestimmung in der Schokolade mit Vorteil verwenden, da sie erheblich einfacher ist, als die bis jetzt gebräuchliche Art der Fettbestimmung.

Es ist noch ein Bedenken zu besprechen, das gegen die oben beschriebeno Methode vorgebracht werden könnte. Bei verhältnismäßig hoher Temperatur wird längere Zeit ein Luftstrom durch das Fett geführt. Es ist die Frage zu beantworten, ob dadurch eine Veränderung in der chemischen Zusammensetzung des Fettes, etwa eine Oxydation der ungasättigten Fettsäuren, eintritt, was eine erhebliche Erniedrigung der Jodzahl herbeiführen würde. Zur Beantwortung dieser Frage habe ich daher aus Kakao der Firma L. Schaal \& Co. in Strabburg i. E., einmal Fett durch die bisher übliche Ätherextraktion nach den Vorschriften der "Vereinbarungen" gewonnen und ein anderesmal nach meiner Methode. In beiden Fetten wurden dann gleichzeitig die Jodzahlen nach der v. Hübl'schen Methode nebeneinander bestimmt. Dabei ergab das mit Äther extrahierte Fett die Jodzahl 32,6 und das andere die Jodzahl 32,2: Diese übereinstimmenden Zahlen beweisen, daß eine tiefgreifende Veränderung des Fettes durch das Hindurchleiten des Luftstromes nicht eingetreten war.

\title{
Zum refraktometrisehen Nachweis eines Wasserzusatzes zur Milch.
}

\author{
Von
}

Edwin Ackermann, Kantonschemiker in Genf.

[Eingegangen am 23. September 1908.]

In seinem interessanten Vortrage auf der 7. Jahresversammlung der Freien Vereinigung Dentscher Nahrungsmittelchemiker in Nauheim ${ }^{t}$ ) hat C. Mai die Frage des

1) Diese Zeitschrift 1908, 16, 17. 